\title{
Laparoscopic Diagnosis for Spermatic Cord Metastasis from Esophageal Squamous Cell Carcinoma: Report of a Case
}

\author{
Takeshi Matsutani, Tsutomu Nomura, Nobutoshi Hagiwara, \\ Itsuo Fujita, Yoshikazu Kanazawa, Daisuke Kakinuma, \\ Hitoshi Kanno, Akihisa Matsuda and Eiji Uchida
}

\begin{abstract}
Department of Gastrointestinal and Hepato-Biliary-Pancreatic Surgery, Nippon Medical School
We report a case of previously unreported metastasis to the spermatic cord from esophageal squamous cell carcinoma. A 63-year-old Japanese man underwent laparoscopy-assisted esophageal bypass surgery for an advanced esophageal cancer. An elastic hard tumor was found in the right lateral inguinal fossa on intraoperative laparoscopy, and laparoscopic enucleation was performed. Histological examination of the resected tumor revealed a moderately differentiated squamous cell carcinoma, compatible with metastasis from the esophageal cancer. Two months after the operation, computed tomography revealed a heterogeneously enhanced groin mass $(20 \mathrm{~mm}$ in diameter) involving the right spermatic cord. To our knowledge, this is the first reported case of a solitary metastasis to the spermatic cord from esophageal squamous cell carcinoma. (J Nippon Med Sch 2015; 82: 304-307)
\end{abstract}

Key words: spermatic cord metastatic tumor, esophageal cancer

\section{Introduction}

Metastatic carcinoma of the spermatic cord from gastrointestinal cancer is extremely rare, even less common than primary tumors of the spermatic cord ${ }^{1}$. Accordingly, this condition's clinicopathological characteristics remain unknown. Advanced esophageal squamous-cell carcinomas often metastasize to lymph nodes, the lungs, or the liver, but they metastasize extremely rarely to only the spermatic cord. We describe a case of squamous cell carcinoma of the lower thoracic esophagus that metastasized to the spermatic cord.

\section{Case Presentation}

A 63-year-old man with dysphagia was referred to our hospital to receive treatment for esophageal cancer. Physical examination showed no remarkable findings. The results of hematologic and serum chemical tests were within normal limits. The concentrations of tumor markers were as follows: carcinoembryonic antigen, $2.7 \mathrm{ng} /$ $\mathrm{mL}$ (normal, $0-5 \mathrm{ng} / \mathrm{mL}$ ); squamous cell carcinoma antigen, $3.8 \mathrm{ng} / \mathrm{mL}$ (normal, 0-1.5 ng/mL); and cytokeratin19 fragments, $4.3 \mathrm{ng} / \mathrm{mL}$ (normal, $0-3.5 \mathrm{ng} / \mathrm{mL}$ ). A bar- ium esophagogram showed a severe stricture in the lower thoracic esophagus (Fig. 1a). Upper gastrointestinal endoscopy revealed an ulcerated tumor with infiltration, classified as type 3, in the lower thoracic esophagus (Fig. 1b). Histological examination of biopsy specimens revealed predominantly moderately differentiated squamous cell carcinoma (Fig. 1c). Computed tomography (CT) of the chest and abdomen showed no evidence of metastasis to the liver or lungs or of peritoneal dissemination, expect for metastases to lymph nodes in the perigastric region. Therefore, esophageal carcinoma, clinical stage III (T3N2M0), was diagnosed according to the Japanese Classification of Esophageal Cancer, 10th Edition².

The patient received neoadjuvant chemotherapy with fluorouracil at $600 \mathrm{mg} / \mathrm{m}^{2}$ (days 1-5), docetaxel at 60 $\mathrm{mg} / \mathrm{m}^{2}$ (day 1 ), and cisplatin at $60 \mathrm{mg} / \mathrm{m}^{2}$ (day 1). Clinical evaluation after chemotherapy showed a partial response of the primary esophageal tumor, but subsequent radiographic examination showed progressive disease with metastasis to the right lung.

To treat the remaining clinical stage $\mathrm{IVb}$ esophageal carcinoma, we performed laparoscopy-assisted esopha-

Correspondence to Takeshi Matsutani, MD, Department of Gastrointestinal and Hepato-Biliary-Pancreatic Surgery, Nippon Medical School, 1-1-5 Sendagi, Bunkyo-ku, Tokyo 113-8603, Japan

E-mail: matsutani@nms.ac.jp

Journal Website (http://www.nms.ac.jp/jnms/) 

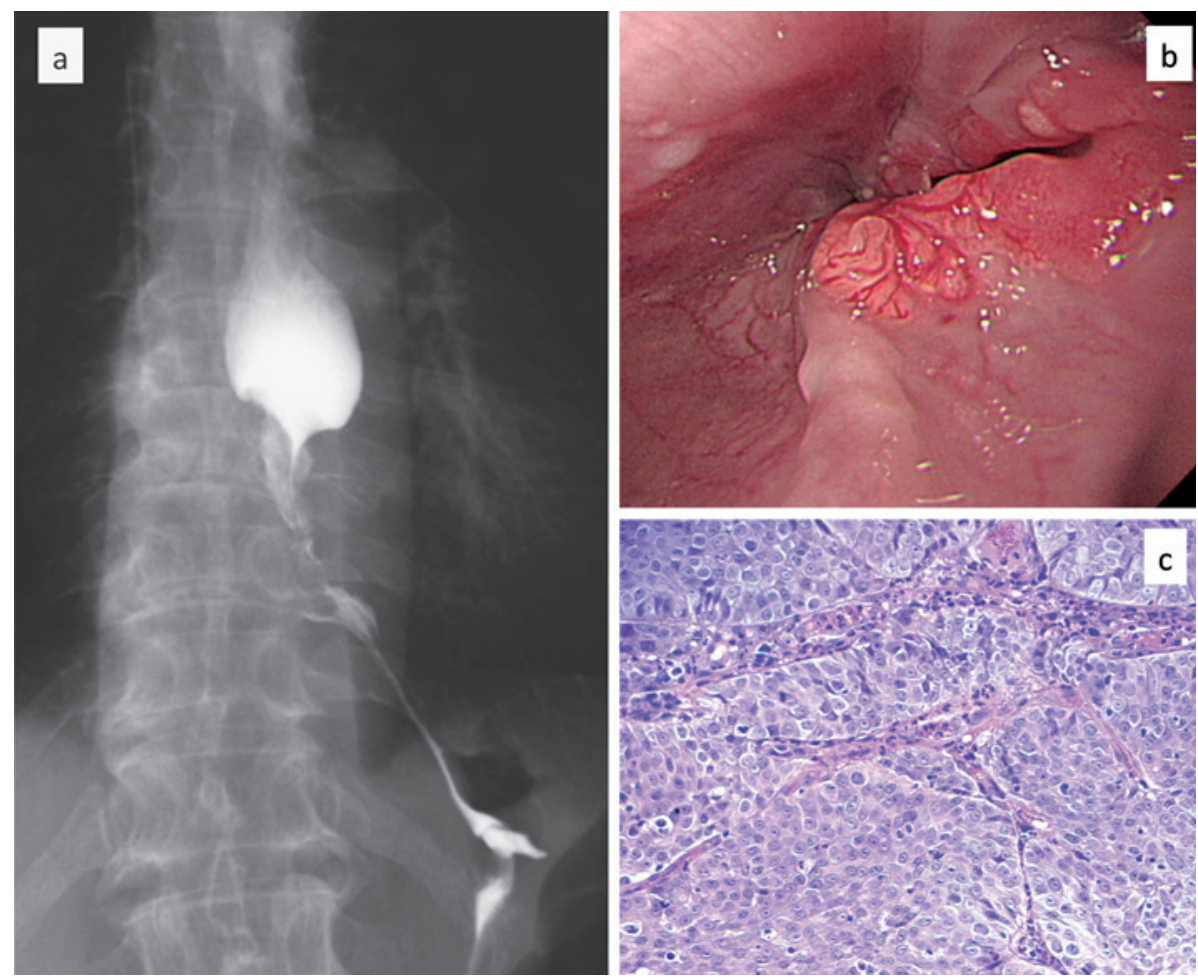

Fig. 1 A barium esophagogram showing a severe stricture in the lower thoracic esophagus (a). Upper gastrointestinal endoscopy reveals an ulcerated tumor with infiltration (b). Histological examination of the biopsy specimens demonstrates moderately differentiated squamous cell carcinoma (c).

geal bypass surgery with the patient under general anesthesia. Briefly, laparoscopy-assisted gastric mobilization was performed, and a gastric tube was made. The esophagus was divided at the neck and abdomen, and the proximal stump was closed. Esophagojejunostomy was performed with a Roux-en-Y anastomosis, and esophagogastrostomy was performed at the neck after the gastric tube had been brought up through the retrosternal route. Intraoperative exploration revealed no peritoneal dissemination. However, an elastic hard tumor was found in the right lateral inguinal fossa without an inguinal hernia or hernia sac (Fig. 2a). Laparoscopic enucleation was performed (Fig. 2b). The laparoscopic and macroscopic findings suggested that the tumor had invaded the region of the spermatic cord. Histological examination of the tumor revealed a moderately differentiated squamous cell carcinoma (Fig. 2c). We therefore concluded that the groin mass was a metastasis from the esophageal squamous cell carcinoma. Two months after the operation, CT demonstrated a heterogeneously enhanced groin mass, $20 \mathrm{~mm}$ in diameter, in the region of the right spermatic cord which had not been detected with CT during the initial surgery; no other metastatic le- sions were found in the pelvis (Fig. 3). The patient received no adjuvant chemotherapy and died of peritoneal dissemination 3 months after surgery.

\section{Discussion}

Malignant lesions of the spermatic cord are extremely rare, and of these tumors, $8.1 \%$ are metastatic carcino$\operatorname{mas}^{3-5}$. The primary carcinomas reported in 1983 to most frequently metastasize to the spermatic cord and epididymis were those of the stomach $(42.8 \%)$ and prostate $(28.5 \%)^{3}$. A review of 13,500 autopsy cases and 641 testicular biopsies and orchiectomies for tumors found that of metastatic tumors of the spermatic cord only $2(0.01 \%)$ were from primary cancers of the stomach ${ }^{1}$. Other reported primary sources of metastases to the spermatic cord include the colon $(28.9 \%)$, the rectum $(7.9 \%)$, the pancreas (15.8\%), the bowel (13.2\%), the bladder, lung, and brain $^{6-9}$. A review of the Japanese literature indicates that gastric adenocarcinoma is the most common source of metastasis to the spermatic cord ${ }^{4}$. However, solitary metastasis of esophageal squamous cell carcinoma to the spermatic cord is extremely rare. A PubMed search we performed of the medical literature found no previously 

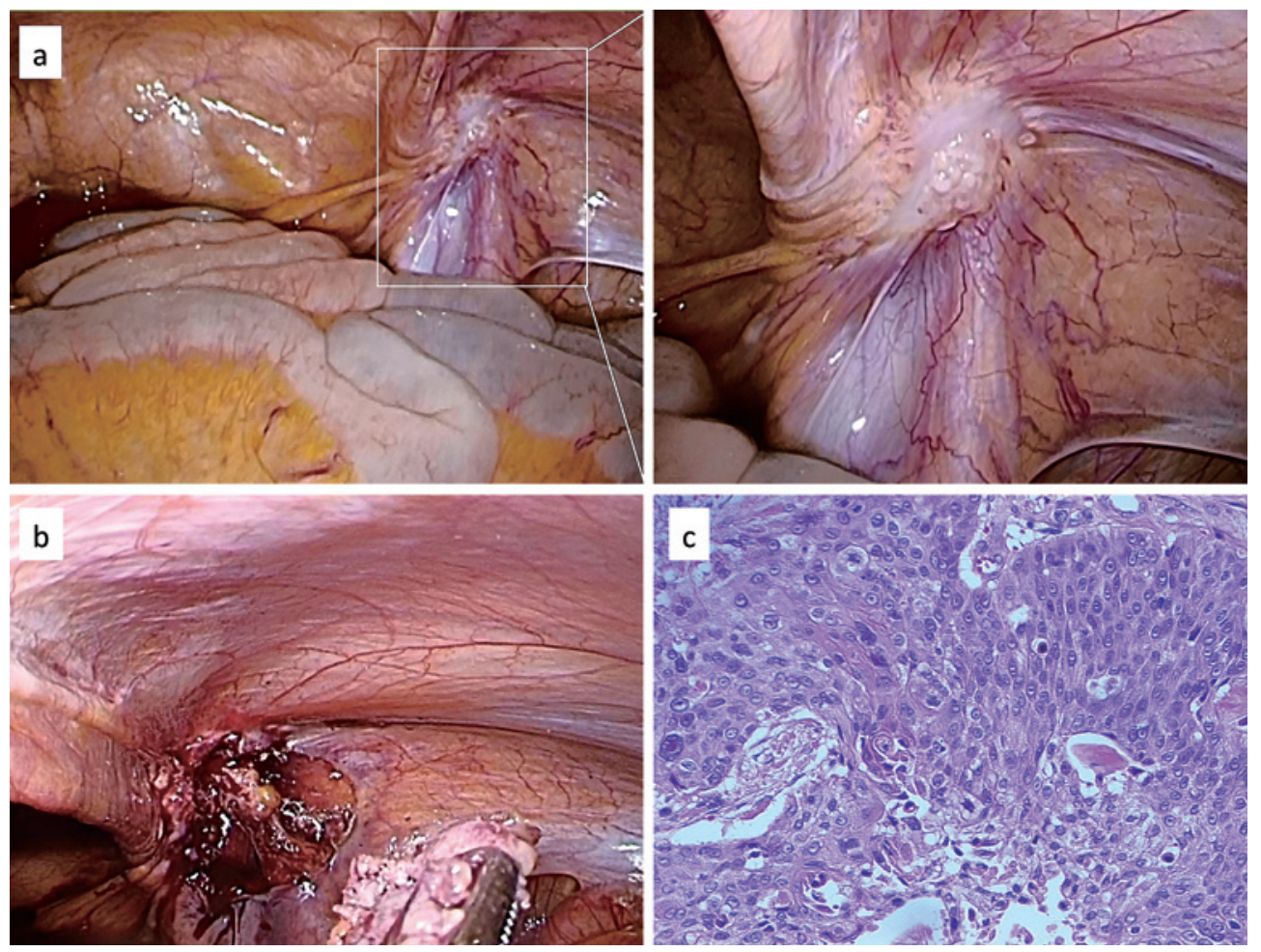

Fig. 2 Intraoperative findings. The tumor is localized in the right lateral inguinal fossa (a). Laparoscopic enucleation is performed (b). Histological examination of the tumor reveals moderately differentiated squamous cell carcinoma (c).

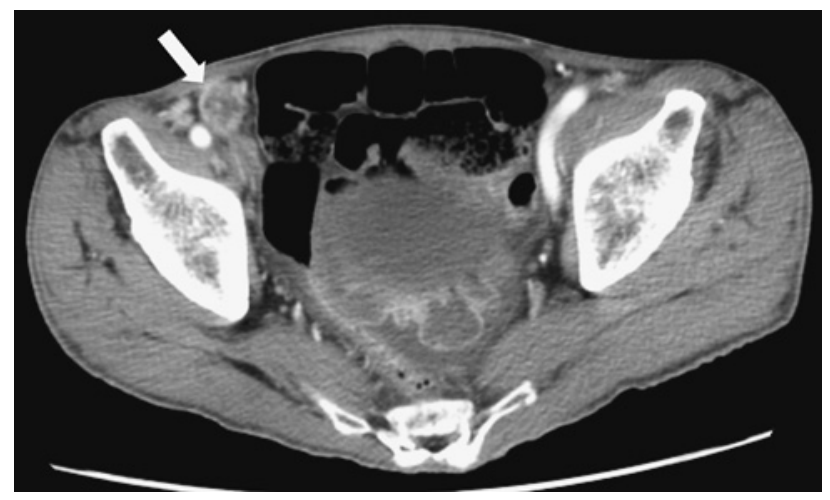

Fig. 3 Follow-up computed tomographic examination demonstrates an enhanced groin mass located in the region of the right spermatic cord.

reported case of solitary metastasis from an esophageal cancer to the spermatic cord. To our knowledge, the present case is the first to be reported.

Most patients with metastatic spermatic cord tumors have a painless scrotal mass, although a lower inguinal mass and enlargement of the testis can also occur. Metastatic spermatic cord tumors are most often misdiagnoses as inguinal hernia, hydrocele, or testis tumor ${ }^{10}$. Spermatic cord tumors generally present as firm, nontender, and nontransluminant masses able to be separated from the testis. Our patient survived for 3 months and did not have right groin pain. Physical examination showed no palpable mass in the right inguinal region or testis.

The exact mechanisms by which primary malignant neoplasms metastasize to the spermatic cord remain unknown; however, several possibilities have been proposed $^{1}$. The main routes are believed to be systemic hematogenous or lymphatic. Other reported routes include retrograde vascular extension and transperitoneal seeding. Histological examination did not demonstrate any specific features directly related to the mechanism of spermatic metastasis. In the present case of metastasis to the spermatic cord, the most likely metastatic pathway was suggested by laparoscopic exploration to be peritoneal metastasis. The lateral inguinal fossa is a shallow depression on the inner aspect of the abdominal wall, situated lateral to the lateral umbilical fold. Cancer cells migrating to the peritoneal cavity might have been trapped in this peritoneal depression and subsequently multiplied.

Gastrointestinal cancer with spermatic cord metastasis is already an advanced disease with an extremely poor prognosis. The mean survival time after diagnosis has been reported to be approximately 9 months ${ }^{3}$. Whether 
such a condition can be beneficially treated with systemic chemotherapy or chemoradiotherapy remains controversial. Although curative resection of metastases might not improve survival, to evaluate the potential benefits of surgery in patients with spermatic cord metastasis additional patients must be studied.

In conclusion, although rare, a solitary metastatic lesion of the spermatic cord arising from esophageal squamous cell carcinoma should be included in the differential diagnosis of a groin mass. Whether the potentially curative resection of such lesions can improve survival remains unclear.

Conflict of Interest: None of the authors have any conflicts of interest to declare.

\section{References}

1. Dutt N, Bates AW, Baithun SI: Secondary neoplasms of the male genital tract with different patterns of involvement in adults and children. Histopathology 2000; 37: 323-331.

2. Japan Esophageal Society: Japan Esophageal Society. Japanese classification of esophageal cancer, tenth edition: part I. Esophagus 2009; 6: 1-25.

3. Algaba F, Santaularia JM, Villavicencio H: Metastatic tu- mor of the epididymis and spermatic cord. Eur Urol 1983; 9: 56-59.

4. Kanno K, Ohwada S, Nakamura S, Ohya T, Iino Y, Morishita Y, Hayashi M, Yamanaka H, Fukusato T, Koyama T, et al.: Epididymis metastasis from colon carcinoma: a case report and a review of the Japanese literature. Jpn J Clin Oncol 1994; 24: 340-344.

5. Kanazawa Y, Kato S, Fujita I, Onodera H, Takata H, Onda M, Naito Z, Uchida E: Spermatic cord tumor metastatic from stomach cancer 1 year after curative gastrectomy. J Nippon Med Sch 2013; 80: 318-323.

6. Issa MM, Kabalin JN, Dietrick DD, Reese J, Freiha FS: Spermatic cord metastasis from transitional cell carcinoma of the bladder. Urology 1994; 43: 561-563.

7. Kaya C, Tanrikulu H, Yilmaz G, Aker F, Karaman MI: Spermatic cord metastasis as an initial manifestation of non-small cell carcinoma of the lung. Int J Urol 2006; 13: 846-848.

8. Meacham RB, Mata JA, Espada R, Wheeler TM, Schum CW, Scardino PT: Testicular metastasis as the first manifestation of colon carcinoma. J Urol 1988; 140: 621-622.

9. Thambi dorai CR, Azmi A, Rahman AJ, Subathra S, Hayati AR, Zulfiqar A: Spermatic cord metastasis from a medulloblastoma. Pediatr Surg Int 2001; 17: 654-656.

10. Arlen M, Grabstald H, Whitmore WF Jr: Malignant tumors of the spermatic cord. Cancer 1969; 23: 525-532.

(Received, September 24, 2015)

(Accepted, November 24, 2015) 